九州大学学術情報リポジトリ

Kyushu University Institutional Repository

Center manifold reduction for large populations of globally coupled phase oscillators

Chiba, Hayato

Institute of Mathematics for Industry, Kyushu University

Nishikawa, Isao

Institute of Industrial Science, University of Tokyo

ht tp://hdl. hand le. net/2324/25695

出版情報: Chaos. 21 (4)，pp.043103(1)-043103(10)，2011-12-12. American Institute of Physics バージョン:

権利関係: (C) 2011 American Institute of Physics 


\title{
Center manifold reduction for large populations of globally coupled phase oscillators
}

\author{
Hayato Chiba $^{1}$ and Isao Nishikawa ${ }^{2}$ \\ ${ }^{1}$ Institute of Mathematics for Industry, Kyushu University, Fukuoka 819-0395, Japan \\ ${ }^{2}$ Institute of Industrial Science, University of Tokyo, Tokyo 153-8505, Japan
}

(Received 7 April 2011; accepted 16 September 2011; published online 7 October 2011)

\begin{abstract}
A bifurcation theory for a system of globally coupled phase oscillators is developed based on the theory of rigged Hilbert spaces. It is shown that there exists a finite-dimensional center manifold on a space of generalized functions. The dynamics on the manifold is derived for any coupling functions. When the coupling function is $\sin \theta$, a bifurcation diagram conjectured by Kuramoto is rigorously obtained. When it is not $\sin \theta$, a new type of bifurcation phenomenon is found due to the discontinuity of the projection operator to the center subspace. (C) 2011 American Institute of Physics. [doi:10.1063/1.3647317]
\end{abstract}

The dynamics of systems of large populations of coupled oscillators have been of great interest because collective synchronization phenomena are observed in a variety of areas. The stability of (de)synchronous states and bifurcations from them are the main issues to understand the behavior of systems. Since usual stability and bifurcation theory in dynamical systems are not applicable to such systems when the dimensions of systems are too large, much work has been done to understand the dynamics. However, except for special simple cases, to investigate bifurcations is a quite difficult problem and much remains unclear. In this paper, a correct bifurcation theory for such systems is proposed by means of the theory of generalized functions, which is applicable to large classes of coupled phase oscillators including the Kuramoto model. To use a space of generalized functions is suitable to study the behavior of statistical quantities such as the order parameter. This will be demonstrated for two cases. For the Kuramoto model, a well known Kuramoto's bifurcation diagram will be rigorously obtained. For a certain system including the second harmonic in the coupling function, a new type of bifurcation phenomenon will be found.

\section{INTRODUCTION}

Collective synchronization phenomena are observed in a variety of areas, such as chemical reactions, engineering circuits, and biological populations. ${ }^{1}$ In order to investigate such phenomena, a system of globally coupled phase oscillators of the following form is often used:

$$
\frac{d \theta_{k}}{d t}=\omega_{k}+\frac{K}{N} \sum_{j=1}^{N} f\left(\theta_{j}-\theta_{k}\right), \quad k=1, \ldots, N,
$$

where $\theta_{k}(t)$ denotes the phase of a $k$-th oscillator, $\omega_{k} \in \mathbf{R}$ denotes its natural frequency drawn from some distribution function $g(\omega), K>0$ is the coupling strength, and $f(\theta)=\sum_{n=-\infty}^{\infty} f_{n} e^{i n \theta}$ is a $2 \pi$-periodic function $(i=\sqrt{-1})$.
When $f(\theta)=\sin \theta$, it is referred to as the Kuramoto model. ${ }^{2}$ In this case, it is numerically observed that if $K$ is sufficiently large, some or all of the oscillators tend to rotate at the same velocity on average, which is referred to as synchronization. ${ }^{1,3}$ In order to evaluate whether synchronization occurs, Kuramoto introduced the order parameter $r(t) e^{i \psi(t)}$, which is given by

$$
r(t) e^{i \psi(t)}:=\frac{1}{N} \sum_{j=1}^{N} e^{i \theta_{j}(t)}
$$

When a synchronous state is formed, $r(t)$ takes a positive value. Indeed, based on some formal calculations, Kuramoto assumed a bifurcation diagram of $r(t)$ : Suppose $N \rightarrow \infty$. If $g(\omega)$ is an even and unimodal function such that $g^{\prime \prime}(0) \neq 0$, then the bifurcation diagram of $r(t)$ is as in Fig. 1(a). In other words, if the coupling strength $K$ is smaller than $K_{c}:=2 /(\pi g(0))$, then $r(t) \equiv 0$ is asymptotically stable. If $K$ exceeds $K_{c}$, then a stable synchronous state emerges. Near the transition point $K_{c}, r$ is of order $O\left(\left(K-K_{c}\right)^{1 / 2}\right)$. See Ref. 3 for Kuramoto's discussion.

In the last two decades, several studies have been performed in an attempt to confirm Kuramoto's assumption. Daido $^{4}$ calculated steady states of Eq. (1) for any $f$ using an argument similar to Kuramoto's. Although he obtained various bifurcation diagrams, the stability of solutions was not demonstrated. In order to investigate the stability of steady states, Strogatz and Mirollo and coworker ${ }^{5-8}$ performed a linearized analysis. The linear operator $T_{1}$, which is obtained by linearizing the Kuramoto model, has a continuous spectrum on the imaginary axis. Nevertheless, they found that the steady states can be asymptotically stable because of the existence of resonance poles on the left half plane. ${ }^{8}$ Since the results of Strogatz and Mirollo and coworker are based on the linearized analysis, the effects of nonlinear terms are neglected. However, investigating nonlinear bifurcations is more difficult because $T_{1}$ has a continuous spectrum on the imaginary axis, that is, a center manifold in the usual sense is of infinite dimension. In order to avoid this difficulty, 

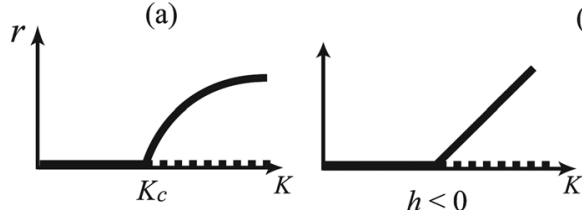

(b)

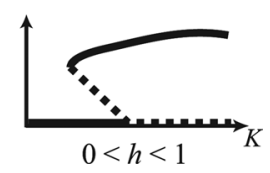

FIG. 1. Bifurcation diagrams of the order parameter for (a) $f(\theta)=\sin \theta$ and (b) $f(\theta)=\sin \theta+h \sin 2 \theta$. The solid lines denote stable solutions, and the dotted lines denote unstable solutions.

Crawford and Davies ${ }^{9}$ added noise of strength $D>0$ to the Kuramoto model. The continuous spectrum then moves to the left side by $D$, and thus, the usual center manifold reduction is applicable. However, their method is not valid when $D=0$. An eigenfunction of $T_{1}$ associated with a center subspace diverges as $D \rightarrow 0$ because an eigenvalue on the imaginary axis is embedded in the continuous spectrum as $D \rightarrow 0$. Recently, Ott and Antonsen ${ }^{10}$ found a special solution of the Kuramoto model, which allows the dimension of the system to be reduced. Their method is applicable only for $f(\theta)=\sin \theta$ because their method relies on a certain symmetry of the system. ${ }^{11}$ Furthermore, the reduced system is still of infinite dimension, except for the case in which $g(\omega)$ is a rational function. Pikovsky and Rosenblum ${ }^{12}$ proposed the reduction of the system based on the construction of constants of motion. Their method also relies on a special form of the system. Thus, a unified bifurcation theory for globally coupled phase oscillators is required.

In the present paper, a correct center manifold reduction is proposed by means of the theory of generalized functions, which is applicable for any coupling function $f$. It is shown that there exists a finite-dimensional center manifold on a space of generalized functions, despite the fact that the continuous spectrum lies on the imaginary axis. This will be demonstrated for two cases, (I) $f(\theta)=\sin \theta$ and (II) $f(\theta)=\sin \theta$ $+h \sin 2 \theta, h \in \mathbf{R}$, and two distribution functions $g(\omega)$, (a) a Gaussian distribution and (b) a rational function (e.g., Lorentzian distribution $\left.g(\omega)=1 /\left(\pi\left(1+\omega^{2}\right)\right)\right)$. For (I), we rigorously prove Kuramoto's conjecture; that is, it is shown for the continuous limit that when $0<K<K_{c}$, the de-synchronous state $r \equiv 0$ is locally asymptotically stable, and when $K_{c}<K$, a stable synchronous state $r>0$ bifurcates from the trivial solution with the order $O\left(\left(K-K_{c}\right)^{1 / 2}\right)$. For (II), a different bifurcation diagram will be obtained, as was predicted by Daido. ${ }^{4}$ The different bifurcation structure is shown to be caused by the discontinuity of the projection to the generalized center subspace. All omitted proofs are given in Ref. 13.

\section{THE CONTINUOUS MODEL}

Let us derive the continuous model (the continuum limit) of Eq. (1) to describe the situation $N \rightarrow \infty$. By introducing the Daido's order parameter ${ }^{4}$

$$
\hat{\eta}_{k}(t):=\frac{1}{N} \sum_{j=1}^{N} e^{i k \theta_{j}(t)}
$$

Equation (1) is rewritten as

$$
\frac{d \theta_{k}}{d t}=\omega_{k}+K \sum_{l=-\infty}^{\infty} f_{i} \hat{\eta}_{l}(t) e^{-i l \theta_{k}}
$$

This implies that the flow of $\theta_{k}$ is generated by the vector field

$$
\hat{v}=\omega_{k}+K \sum_{l=-\infty}^{\infty} f_{l} \hat{\eta}_{l}(t) e^{-i l \theta_{k}}
$$

Hence, we define the continuous model of Eq. (1) as the equation of continuity

$$
\left\{\begin{array}{l}
\frac{\partial \rho_{t}}{\partial t}+\frac{\partial}{\partial \theta}\left(\rho_{t} v\right)=0, \\
v:=\omega+K \sum_{l=-\infty}^{\infty} f_{l} \eta_{l}(t) e^{-i l \theta},
\end{array}\right.
$$

where $\eta_{l}$ is defined to be

$$
\eta_{l}(t)=\int_{\mathbf{R}} \int_{0}^{2 \pi} e^{i l \theta} \rho_{t}(\theta, \omega) g(\omega) d \theta d \omega
$$

$g(\omega)$ is a given probability density function for natural frequencies, and the unknown function $\rho_{t}=\rho_{t}(\theta, \omega)$ is a probability measure on $[0,2 \pi)$ parameterized by $t, \omega \in \mathbf{R}$. In particular, $\eta_{1}$ is a continuous version of Kuramoto's order parameter. Roughly speaking, $\rho_{t}(\theta, \omega)$ denotes a probability that an oscillator having a natural frequency $\omega$ is placed at a position $\theta$.

$$
\begin{aligned}
& \text { Setting } Z_{j}(t, \omega):=\int_{0}^{2 \pi} e^{i j \theta} \rho_{t}(\theta, \omega) d \theta \text { yields } \\
& \qquad \frac{d Z_{j}}{d t}=i j \omega Z_{j}+i j K f_{j} \eta_{j}+i j K \sum_{l \neq j} f_{i} \eta_{l} Z_{j-l} .
\end{aligned}
$$

The trivial solution $Z_{j} \equiv 0(j= \pm 1, \pm 2, \ldots)$ corresponds to the uniform distribution $\rho_{t} \equiv 1 / 2 \pi$ on a circle, which implies $r \equiv 0$ (de-synchronous state). To investigate the stability of this state, we consider the linearized system. Let $L^{2}(\mathbf{R}, g(\omega) d \omega)$ be the weighted Lebesgue space with the inner product

$$
(\psi, \phi)=\int_{\mathbf{R}} \overline{\psi(\omega)} \phi(\omega) g(\omega) d \omega .
$$

Put $P_{0}(\omega) \equiv 1$. Then, the order parameters are written as

$$
\eta_{j}(t)=\int_{\mathbf{R}} Z_{j}(t, \omega) g(\omega) d \omega=\left(\overline{Z_{j}}, P_{0}\right)=\left(P_{0}, Z_{j}\right),
$$

and the linearized system of Eq. (4) is given by

$$
\frac{d Z_{j}}{d t}=T_{j} Z_{j}:=i j \omega Z_{j}+i j K f_{j}\left(P_{0}, Z_{j}\right) .
$$

Let us consider the spectra of linear operators $T_{j}$. The spectrum of $T_{j}$ consists of the continuous spectrum and eigenvalues. The continuous spectrum is the whole imaginary axis. Eigenvalues $\lambda$ of $T_{j}$ are given as roots of the equation

$$
\int_{\mathbf{R}} \frac{1}{\lambda-i j \omega} g(\omega) d \omega=\frac{1}{i j K f_{j}} .
$$

Indeed, the equation $\left(\lambda-T_{j}\right) v=0$ provides

$$
v+i j K f_{j}\left(P_{0}, v\right)(\lambda-i j \omega)^{-1} P_{0}=0 .
$$


Taking the inner product with $P_{0}$, we obtain Eq. (7). It is known that there exists a positive constant $K_{c}^{(j)}$ such that if $K_{c}^{(j)}<K, T_{j}$ has eigenvalues on the right half plane, so that the $j$-th Fourier component of the de-synchronous state is unstable, while if $0<K<K_{c}^{(j)}, T_{j}$ has no eigenvalues. In particular, when $g(\omega)$ is even and unimodal, there exists a unique eigenvalue $\lambda=\lambda_{0}(K)$ on the right half plane for $K_{c}^{(j)}<K$. As $K$ decreases, $\lambda_{0}(K)$ moves to the left, and at $K=K_{c}^{(j)}, \lambda_{0}(K)$ is absorbed into the continuous spectrum on the imaginary axis. In this manner, the eigenvalue suddenly disappears at $K=K_{c}^{(j)}$. For example, if $f$ is an odd function and if $g$ is even and unimodal, then $K_{c}^{(j)}$ is given by

$$
K_{c}^{(j)}=-\frac{\operatorname{Im}\left(f_{j}\right)}{\pi\left|f_{j}\right|^{2} g(0)} \text {. }
$$

In the present paper, for simplicity, we assume that $K_{c}^{(1)}$ is the least number among $K_{c}^{(j)} ; K_{c}:=\inf _{j} K_{c}^{(j)}=K_{c}^{(1)}$. This means that the first Fourier component of the coupling function $f$ is the most dominant term (for $f(\theta)=\sin \theta+h \sin 2 \theta$, which is true if and only if $h<1$ ). When $0<K<K_{c}, T_{j}$ has no eigenvalues and the spectrum consists only of the continuous spectrum on the imaginary axis for any $j$. Thus, the dynamics of the linearized system $d Z_{j} / d t=T_{j} Z_{j}$ is quite nontrivial. It is well known for a finite dimensional system that the trivial solution is neutrally stable if the spectrum lies on the imaginary axis. For infinite dimensional systems, this is not true. Indeed, numerical simulation ${ }^{8,14}$ suggests that the order parameter decays exponentially to zero when $0<K<K_{c}$. Strogatz and coworkers ${ }^{8}$ found that such an exponential decay of the order parameter is induced by a resonance pole, and it is rigorously proved by Chiba. ${ }^{13}$ In Ref. 13 , the spectral theory on rigged Hilbert spaces is developed to reveal the dynamics of the linearized system.

\section{A RIGGED HILBERT SPACE}

A rigged Hilbert space consists of three spaces $X \subset L^{2}(\mathbf{R}, g(\omega) d \omega) \subset X^{\prime}:$ a space $X$ of test functions, a Hilbert space $L^{2}(\mathbf{R}, g(\omega) d \omega)$, and the dual space $X^{\prime}$ of $X$ (a space of continuous anti-linear functionals on $X$, each element of which is referred to as a generalized function). We use Dirac's notation, where for $\mu \in X^{\prime}$ and $\phi \in X, \mu(\phi)$ is denoted by $\langle\phi \mid \mu\rangle$. For $a \in \mathbf{C}$, we have $a\langle\phi \mid \mu\rangle=\langle\bar{a} \phi \mid \mu\rangle=\langle\phi \mid a \mu\rangle$. A usual function can be regarded as a generalized function using an integral kernel. To be precise, the canonical inclusion $i: L^{2}(\mathbf{R}, g(\omega) d \omega) \rightarrow X^{\prime}$ is defined as follows. For $\psi \in L^{2}(\mathbf{R}, g(\omega) d \omega)$, we denote $i(\psi)$ by $|\psi\rangle$, which is defined to be

$$
i(\psi)(\phi)=\langle\phi \mid \psi\rangle:=(\phi, \psi)=\int_{\mathbf{R}} \overline{\phi(\omega)} \psi(\omega) g(\omega) d \omega .
$$

By the canonical inclusion, Eq. (4) is rewritten as an evolution equation on the dual space $X^{\prime}$ as

$$
\frac{d}{d t}\left|Z_{j}\right\rangle=T_{j}^{\times}\left|Z_{j}\right\rangle+i j K \sum_{l \neq j} f_{l}\left\langle P_{0} \mid Z_{l}\right\rangle \cdot\left|Z_{j-l}\right\rangle,
$$

where $T_{j}^{\times}$is a dual operator of $T_{j}$ defined through $\left\langle\phi \mid T_{j}^{\times} \mu\right\rangle$ $=\left\langle T_{j}^{*} \phi \mid \mu\right\rangle$ for $\mu \in X^{\prime}$ and $\phi \in X$, and $T_{j}^{*}$ is the adjoint operator of $T_{j}$. In particular, for any $\psi \in L^{2}(\mathbf{R}, g(\omega) d \omega)$, we have $\left\langle\phi \mid T_{j}^{\times} \psi\right\rangle=\left\langle T_{j}^{*} \phi \mid \psi\right\rangle=\left(T_{j}^{*} \phi, \psi\right)=\left(\phi, T_{j} \psi\right)$. Thus $T_{j}^{\times}$gives an extension of $T_{j}$ to the dual space.

Here, the strategy for the bifurcation theory of globally coupled phase oscillators is to use the space of generalized functions $X^{\prime}$ rather than a space of usual functions. The reason for this is explained intuitively as follows. If we use the space $L^{2}(\mathbf{R}, g(\omega) d \omega)$ to investigate the dynamics, then the behavior of $\rho_{t}$ itself will be obtained. However, it is neutrally stable because of the conservation law $\int_{0}^{2 \pi} \rho_{t}(\theta, \omega) d \theta=1$. What we would like to know is the dynamics of the moments of $\rho_{t}$, in particular, the order parameter. This suggests that we should use a different topology for the stability of $\rho_{t}$. (Note that the definition of stability depends on definition of the topology.) For the purpose of the present study, $\rho_{t}$ is said to be convergent to $\hat{\rho}$ as $t \rightarrow \infty$ if and only if

$$
\begin{aligned}
& \int_{\mathbf{R}} \int_{0}^{2 \pi} \phi(\omega) e^{i j \theta} g(\omega) d \rho_{t}(\theta, \omega), \\
\rightarrow & \int_{\mathbf{R}} \int_{0}^{2 \pi} \phi(\omega) e^{i j \theta} g(\omega) d \hat{\rho}(\theta, \omega),
\end{aligned}
$$

for any $j \in \mathbf{Z}$ and $\phi \in X$. This implies that for the Fourier coefficients, a function $Z_{j}(t, \omega) \in L^{2}(\mathbf{R}, g(\omega) d \omega)$ is said to be convergent to $\hat{Z}_{j}(\omega)$ as $t \rightarrow \infty$ if and only if

$$
\int_{\mathbf{R}} \phi(\omega) Z_{j}(t, \omega) g(\omega) d \omega \rightarrow \int_{\mathbf{R}} \phi(\omega) \hat{Z}_{j}(\omega) g(\omega) d \omega,
$$

for any $\phi \in X$. In other words, $Z_{j}(t, \omega)$ converges to $\hat{Z}_{j}(\omega)$ if and only if $\left\langle\phi \mid Z_{j}\right\rangle \rightarrow\left\langle\phi \mid \hat{Z}_{j}\right\rangle$ for any $\phi \in X$ if we regard $Z_{j}$ as a generalized function. The topology induced by this convergence is referred to as the weak topology. By the completion of $L^{2}(\mathbf{R}, g(\omega) d \omega)$ with respect to the weak topology, we obtain a space of generalized functions $X^{\prime}$. On the space $X^{\prime}$, a function $Z_{1}(t, \omega)$ converges as $t \rightarrow \infty$ if and only if $\left\langle\phi \mid Z_{1}\right\rangle$ converges for any $\phi \in X$. Since the order parameter is written as $\eta_{1}(t)=\left\langle P_{0} \mid Z_{1}\right\rangle$, this topology is suitable for the purpose of the present study. Although a solution of the linearized system $d Z_{j} / d t=T_{j} Z_{j}$ is neutrally stable in $L^{2}(\mathbf{R}$, $g(\omega) d \omega)$-sense because of the continuous spectrum on the imaginary axis, we will show that it can decay to zero exponentially if we use the weak topology. More generally, a rigged Hilbert space provides strong tools for studies of dynamics of moments of measures or probability density functions. A suitable choice of the space $X$ depends on a problem, which will be explained in Sec. IV.

A rigged Hilbert space was introduced by Gelfand ${ }^{15}$ to generalize the theory of Schwartz distributions. See Ref. 16 for a review of Gelfand's work and its application to quantum mechanics.

\section{A SPECTRAL THEORY ON RIGGED HILBERT SPACES}

We give a summary of the spectral theory on rigged Hilbert spaces developed in Ref. 13. In what follows, put $f_{1}=1 /(2 i)$ for simplicity (that is, $f(\theta)=\sin \theta+$ (higher harmonics)) and we consider the operator 


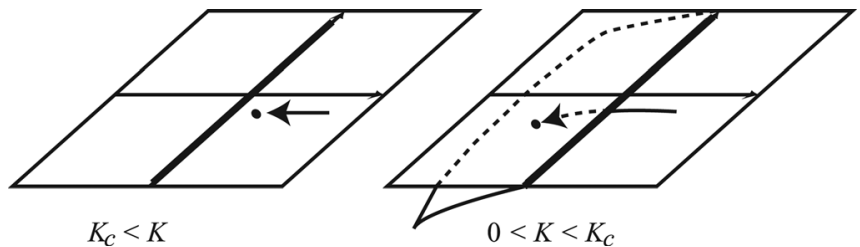

FIG. 2. The motion of the generalized eigenvalue as $K$ decreases. When $K>K_{c}$, it exists on the right half plane as an eigenvalue of $T_{1}$. At $K=K_{c}$, it is absorbed into the continuous spectrum and disappears from the complex plane. When $K<K_{c}$, it goes to the second Riemann sheet of the generalized resolvent and turns into a resonance pole, which is no longer an eigenvalue of $T_{1}$ in the usual sense.

$$
T_{1} \phi=i \omega \phi+\frac{K}{2}\left(P_{0}, \phi\right) P_{0}
$$

Let $e^{T_{1} t} \phi$ be the solution of the linearized system $d Z_{1} / d t=T_{1} Z_{1}$ with the initial condition $\phi(\omega)$. The linear operator $e^{T_{1} t}$ is called the semigroup (semiflow) generated by $T_{1}$. It is known that the semigroup $e^{T_{1} t}$ is given by the Laplace inversion formula

$$
e^{T_{1} t}=\lim _{y \rightarrow \infty} \frac{1}{2 \pi i} \int_{x-i y}^{x+i y} e^{\lambda t}\left(\lambda-T_{1}\right)^{-1} d \lambda
$$

for $t>0$, where $x>0$ is chosen so that the contour is to the right of the spectrum of $T_{1}$ (see Fig. 3(a)). The operator $\left(\lambda-T_{1}\right)^{-1}$ is called the resolvent in functional analysis (in engineering, it is called the Laplace transform). The resolvent is a continuous operator on $L^{2}(\mathbf{R}, g(\omega) d \omega)$ when $\lambda$ does not lie on the spectrum of $T_{1}$. Since $T_{1}$ has the continuous spectrum on the imaginary axis, the inner product $\left(\phi,\left(\lambda-T_{1}\right)^{-1} \psi\right)$ for $\psi, \phi \in L^{2}(\mathbf{R}, g(\omega) d \omega)$ diverges when $\lambda \in i \mathbf{R}$. However, it is possible that $\left(\phi,\left(\lambda-T_{1}\right)^{-1} \psi\right)$ exists on the imaginary axis and has an analytic continuation from the right half plane to the left half plane if $\psi$ and $\phi$ satisfy suitable conditions. To see this idea, it is sufficient to consider the multiplication operator $i \mathcal{M} \phi:=i \omega \phi$; that is, $K=0$ for $T_{1}$. The resolvent is given by

$$
\left(\phi,(\lambda-i \mathcal{M})^{-1} \psi\right)=\int_{\mathbf{R}} \frac{1}{\lambda-i \omega} \psi(\omega) \overline{\phi(\omega)} g(\omega) d \omega,
$$

for any $\psi, \phi \in L^{2}(\mathbf{R}, g(\omega) d \omega)$. In general, the integral in the right hand side diverges as $\operatorname{Re}(\lambda) \rightarrow 0$ because of the factor $1 /(\lambda-i \omega)$. However, if $\psi$ and $\phi$ are holomorphic on the real

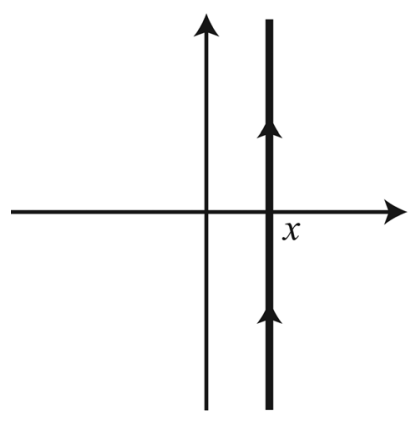

(a)

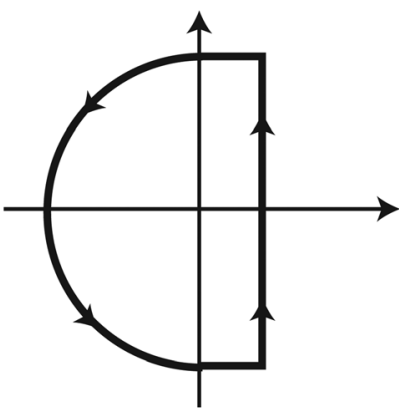

(b)
FIG. 3. The contour for the Laplace inversion formula. axis and the upper half plane, the integral converges as $\operatorname{Re}(\lambda) \rightarrow+0$ and has an analytic continuation from the right half plane to the left half plane, which is given by

$$
\begin{aligned}
& \int_{\mathbf{R}} \frac{1}{\lambda-i \omega} \psi(\omega) \overline{\phi(\omega)} g(\omega) d \omega \\
& \quad+2 \pi \psi(-i \lambda) \overline{\phi(-i \lambda)} g(-i \lambda), \quad \operatorname{Re}(\lambda)<0 .
\end{aligned}
$$

Let $X$ be a vector space consisting of some class of holomorphic functions defined near the upper half plane. The above calculation implies that $\left(\phi,(\lambda-i \mathcal{M})^{-1} \psi\right)$ is an entire function when $\psi, \phi \in X$. The second term of the above quantity is not written as an inner product of two functions. This suggests that the analytic continuation of the resolvent is no longer included in $L^{2}(\mathbf{R}, g(\omega) d \omega)$. Since the complex number $\left(\phi,(\lambda-i \mathcal{M})^{-1} \psi\right)$ exists for each $\phi \in X$, we can regard $(\lambda-i \mathcal{M})^{-1} \psi$ as a functional on $X$; that is, an element of the dual space $X^{\prime}$. To be precise, define the generalized resolvent $A(\lambda)$ of $i \mathcal{M}$ to be

$$
\begin{aligned}
& \langle\phi \mid A(\lambda) \psi\rangle \\
& = \begin{cases}\left(\phi,(\lambda-i \mathcal{M})^{-1} \psi\right) & (\operatorname{Re}(\lambda)>0), \\
\left(\phi,(\lambda-i \mathcal{M})^{-1} \psi\right) & \\
+2 \pi \psi(-i \lambda) \overline{\phi(-i \lambda)} g(-i \lambda) & (\operatorname{Re}(\lambda)<0) .\end{cases}
\end{aligned}
$$

Since the mapping $\phi \mapsto\langle\phi \mid A(\lambda) \psi\rangle$ is linear, $|A(\lambda) \psi\rangle$ is an element of $X^{\prime}$. Since $|A(\lambda) \psi\rangle$ is determined for each $\psi \in X$, $A(\lambda)$ is a linear operator from $X$ into $X^{\prime}$ and is analytic with respect to $\lambda \in \mathbf{C}$. By the definition, $A(\lambda) \psi=(\lambda-i \mathcal{M})^{-1} \psi$ when $\operatorname{Re}(\lambda)>0$. Now, we have shown that the resolvent $(\lambda-i \mathcal{M})^{-1}$ as an operator on $L^{2}(\mathbf{R}, g(\omega) d \omega)$ diverges on the imaginary axis, however, if we regard it as an operator from $X$ into $X^{\prime}$, it has an analytic continuation, which is denoted by $A(\lambda)$, from the right half plane to the left half plane.

Similarly, we can prove that the resolvent $\left(\lambda-T_{1}\right)^{-1}$ of the operator $T_{1}$ does not exist as an operator on $L^{2}(\mathbf{R}$, $g(\omega) d \omega)$ when $\lambda \in i \mathbf{R}$. If we regard it as an operator from $X$ into $X^{\prime}$, however, it has a meromorphic continuation $\mathcal{R}_{\lambda}$ from the right half plane to the left half plane. Put $\mathcal{P} \phi=\frac{K}{2}\left(P_{0}, \phi\right)$. Then, $T_{1}=i \mathcal{M}+\mathcal{P}$. The resolvent of $T_{1}$ is rewritten as

$$
\left(\lambda-T_{1}\right)^{-1}=(\lambda-i \mathcal{M})^{-1} \circ\left(i d-\mathcal{P}(\lambda-i \mathcal{M})^{-1}\right)^{-1},
$$

where id is the identity mapping. Hence, the analytic continuation of $\left(\lambda-T_{1}\right)^{-1}$ in the generalized sense is given by

$$
\mathcal{R}_{\lambda}:=A(\lambda) \circ\left(i d-\mathcal{P}^{\times} A(\lambda)\right)^{-1},
$$

which is a linear operator from $X$ into $X^{\prime}$.

The generalized resolvent $\mathcal{R}_{\lambda}$ is a meromorphic function with respect to $\lambda$. A pole of the generalized resolvent is called a generalized eigenvalue. A point $\lambda$ is a generalized eigenvalue if and only if the operator $i d-\mathcal{P}^{\times} A(\lambda)$ is not injective on $X$. For $T_{1}$, generalized eigenvalues are given as roots of the equation

$$
\int_{\mathbf{R}} \frac{1}{\lambda-i \omega} g(\omega) d \omega=\frac{2}{K}, \quad(\operatorname{Re}(\lambda)>0),
$$




$$
\int_{\mathbf{R}} \frac{1}{\lambda-i \omega} g(\omega) d \omega+2 \pi g(-i \lambda)=\frac{2}{K}, \quad(\operatorname{Re}(\lambda)<0) .
$$

In particular, a root of Eq. (13) is called the resonance pole. Note that Eq. (12) is the same as Eq. (7) (for $j=1$, $\left.f_{1}=1 /(2 i)\right)$. Thus, generalized eigenvalues on the right half plane are eigenvalues of $T_{1}$ in the usual sense. Recall that Eq. (12) has a root if and only if $K>K_{c}$. In particular, when $g(\omega)$ is even and unimodal, there exists a unique eigenvalue $\lambda=\lambda_{0}(K)$ on the right half plane for $K>K_{c}$. As $K \rightarrow K_{c}+0$, the eigenvalue $\lambda_{0}(K)$ is absorbed into the continuous spectrum on the imaginary axis and disappears. However, it is easy to show that even for $0<K<K_{c}, \lambda_{0}(K)$ remains to exist as a root of Eq. (13) because the left hand side of Eq. (13) is an analytic continuation of that of Eq. (12). This means that although $\lambda_{0}(K)$ disappears from the original complex plane at $K=K_{c}$, it still exists for $0<K<K_{c}$ as a resonance pole on the Riemann surface of the generalized resolvent $\mathcal{R}_{\lambda}$ (see Fig. 2). We will show that resonance poles induce an exponential decay of a solution.

Let $\lambda_{0}, \lambda_{1}, \lambda_{2}, \ldots$ be the generalized eigenvalues of $T_{1}$ and $T_{1}^{\times}$a dual operator of $T_{1}$. We can show that there exist generalized functions $\mu_{j} \in X^{\prime}$ such that $T_{1}^{\times}\left|\mu_{j}\right\rangle=\lambda_{j}\left|\mu_{j}\right\rangle$; that is, generalized eigenvalues are indeed eigenvalues of the dual operator. The generalized eigenfunction $\mu_{j}$ associated with $\lambda_{j}$ is given by

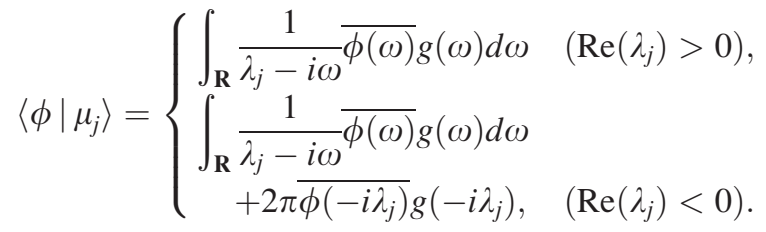

Now that the analytic continuation $\mathcal{R}_{\lambda}$ of the resolvent $\left(\lambda-T_{1}\right)^{-1}$ and its poles are obtained, the Laplace inversion formula (11) can be calculated by using the residue theorem. Considering the space $L^{2}(\mathbf{R}, g(\omega) d \omega), T_{1}$ has the continuous spectrum on the imaginary axis, and thus we cannot deform the contour of the Laplace inversion formula toward the left half plane. However, if we regard the resolvent as an operator from $X$ into $X^{\prime}$, the integrand of the Laplace inversion formula has an analytic continuation to the left half plane. Indeed, the semigroup is rewritten as

$$
\begin{aligned}
\left(\phi, e^{T_{1} t} \psi\right) & =\frac{1}{2 \pi i} \int_{x-i \infty}^{x+i \infty} e^{\lambda t}\left(\phi,\left(\lambda-T_{1}\right)^{-1} \psi\right) d \lambda, \\
& =\frac{1}{2 \pi i} \int_{x-i \infty}^{x+i \infty} e^{\lambda t}\left\langle\phi \mid \mathcal{R}_{\lambda} \psi\right\rangle d \lambda,
\end{aligned}
$$

for $\psi, \phi \in X$. Generalized eigenvalues are poles of $\left\langle\phi \mid \mathcal{R}_{\lambda} \psi\right\rangle$. In the following, for simplicity, we assume that all $\lambda_{n}$ 's are single roots (this is true when $g(\omega)$ is the Gaussian). By deforming the contour as is shown in Fig. 3(b), we can prove the equality

$$
\left(\phi, e^{T_{1} t} \psi\right)=\sum_{n=0}^{\infty} \frac{K}{2 D_{n}} e^{\lambda_{n} t}\left\langle\phi \mid \mu_{n}\right\rangle\left\langle\bar{\psi} \mid \mu_{n}\right\rangle,
$$

where the constant $D_{n}$ is defined by

$$
D_{n}=\lim _{\lambda \rightarrow \lambda_{n}} \frac{1}{\lambda-\lambda_{n}}\left(1-\frac{K}{2} \int_{\mathbf{R}} \frac{g(\omega)}{\lambda-i \omega} d \omega-\pi K g(-i \lambda)\right),
$$

which arises from the residue around $\lambda_{n}$. Since $\left(\phi, e^{T_{1} t} \psi\right)=\left\langle\phi \mid\left(e^{T_{1} t}\right)^{\times} \psi\right\rangle$, the above equality is also written as the equality on the dual space $X^{\prime}$ as

$$
\left(e^{T_{1} t}\right)^{\times}|\psi\rangle=\sum_{n=0}^{\infty} \frac{K}{2 D_{n}} e^{\lambda_{n} t}\left\langle\bar{\psi} \mid \mu_{n}\right\rangle \cdot\left|\mu_{n}\right\rangle,
$$

for any $\psi \in X$. This gives the spectral decomposition of the dual operator of $e^{T_{1} t}$ using the generalized functions.

Recall that the space $X$ consists of holomorphic functions defined near the upper half plane so that the analytic continuation of the resolvent exists. Further, we should choose $X$ so that the integral of the Laplace inversion formula along the contour shown in Fig. 3(b) converges for any $\psi, \phi \in X$ as the radius of the arc tends to infinity. A suitable choice of $X$ and the distribution of resonance poles depend on $g(\omega)$.

(a) When $g(\omega)$ is a Gaussian distribution, let $\operatorname{Exp}_{+}(\beta)$ be the set of holomorphic functions defined near the upper half plane such that $|\phi(z)| e^{-\beta|z|}$ is bounded on the real axis and the upper half plane. Set $X=\operatorname{Exp}_{+}:=\bigcup_{\beta \geq 0} \operatorname{Exp}_{+}(\beta)$. We can introduce a suitable topology on $\operatorname{Exp}_{+}$so that the dual space $\operatorname{Exp}_{+}^{\prime}$ becomes a complete metric space, which allows the existence of a center manifold on $\operatorname{Exp}_{+}^{\prime}$ to be proven. Since the analytic continuation of $g(\omega)$ is a transcendental entire function, infinity $\infty$ is an essential singularity, which proves that Eq. (13) has infinitely many roots that accumulate at $\infty$.

(b) When $g(\omega)$ is a rational function, $X:=H_{+}$is a space of bounded holomorphic functions on the real axis and the upper half plane. It is remarkable that when $g(\omega)$ is rational, Eq. (13) is reduced to an algebraic equation, so that the number of resonance poles is finite. Therefore, the right hand side of Eq. (15) is a finite sum. This implies that the semigroup $\left(e^{T_{1} t}\right)^{\times}$behaves as an "exponential of a matrix." This fact is explained by means of the rigged Hilbert space as follows: Although $H_{+}$is an infinite dimensional vector space, its inclusion $i\left(H_{+}\right) \subset H_{+}^{\prime}$ into the dual space becomes a finitedimensional vector space (i.e., the canonical inclusion $i$ is not injective). Hence, if we regard the infinitedimensional system (4) as the system defined on the dual space by the canonical inclusion, Eq. (10) becomes essentially a finite-dimensional system. This is why in Refs. 10 and 17, the system is reduced to a finitedimension system when $g(\omega)$ is the (sum of the) Lorentzian distribution.

Equation (15) completely determines the dynamics of the order parameter for the linearized system. The order parameter of the system $d Z_{1} / d t=T_{1} Z_{1}$ with the initial condition $\psi$ is given as

$$
\eta_{1}(t)=\left(P_{0}, e^{T_{1} t} \psi\right)=\left\langle P_{0} \mid\left(e^{T_{1} t}\right)^{\times} \psi\right\rangle .
$$

If $0<K<K_{c}$, then all generalized eigenvalues $\lambda_{n}$ lie on the left half plane. As a result, $\eta_{1}(t)$ decays to zero exponentially as $t \rightarrow \infty$, which proves the asymptotic stability of the desynchronous state. 
The other operators $T_{2}, T_{3}, \ldots$ are investigated in the same way. Due to the assumption $\inf _{j} K_{c}^{(j)}=K_{c}^{(1)}=K_{c}$, all generalized eigenvalues of $T_{j}(j \neq 1)$ lie on the left half plane when $0<K \leq K_{c}$. Thus $\eta_{j}=\left\langle P_{0} \mid\left(e^{T_{j} t}\right)^{\times} \psi\right\rangle$ decays to zero exponentially as $t \rightarrow \infty$.

\section{CENTER MANIFOLD REDUCTION}

When $K>0$ is sufficiently small, all resonance poles lie on the left half plane. As $K$ increases, they move to the right side, and when $K=K_{c}$, there exist resonance poles (of $T_{1}$ ) on the imaginary axis. It is well known that a bifurcation occurs when an eigenvalue gets across the imaginary axis as a parameter varies. Let us show that a bifurcation also occurs when a resonance pole gets across the imaginary axis.

For each $j,\left|Z_{j}\right\rangle$ is an element of the dual space $X^{\prime}$. Let $X_{j}^{\prime}(j= \pm 1, \pm 2, \cdots)$ be copies of $X^{\prime}$. We regard the system Eq. (10) as an evolution equation on $\prod_{j \neq 0} X_{j}^{\prime}$. The generalized center subspace $\mathbf{E}_{c}$ is defined as a space spanned by generalized eigenfunctions associated with resonance poles on the imaginary axis, say $\lambda_{0}, \ldots, \lambda_{M}$, which exist only when $K=K_{c}$. Since resonance poles of $T_{j}(j \neq 1)$ lie on the left half plane, $\mathbf{E}_{c} \subset X_{1}^{\prime}$. Equation (15) suggests that the projection $\Pi_{c}$ to $\mathbf{E}_{c}$ is given by

$$
\Pi_{c}|\psi\rangle=\sum_{n=0}^{M} \frac{K}{2 D_{n}}\left\langle\bar{\psi} \mid \mu_{n}\right\rangle \cdot\left|\mu_{n}\right\rangle
$$

Unfortunately, $\Pi_{c}: X \subset X^{\prime} \rightarrow X^{\prime}$ is not continuous because the topology on $X^{\prime}$ is too weak; when $|\psi\rangle \rightarrow 0$ in $X^{\prime}, \Pi_{c}|\psi\rangle$ does not tend to zero in general. When $X=\operatorname{Exp}_{+}=\bigcup_{\beta \geq 0} \operatorname{Exp}_{+}(\beta)$, it is proven in Ref. 13 that $\Pi_{c}$ becomes a continuous operator if the domain is restricted to the subspace $i\left(\operatorname{Exp}_{+}(0)\right) \subset X^{\prime}$. For a solution of Eq. (4), $Z_{1}, Z_{2}, \ldots$ are included in $\operatorname{Exp}_{+}(0)$ (if initial conditions are), although $Z_{-1}, Z_{-2}, \ldots$ are not. To see this, again it is sufficient to consider the case $K=0$. In this case, $T_{j} \phi=i j \omega \phi$ and the solution of the equation $d Z_{j} / d t=T_{j} Z_{j}$ with the initial condition $\phi \in \operatorname{Exp}_{+}(0)$ is given by $Z_{j}(t, \omega)=e^{i j \omega t} \phi(\omega)$. When $j \geq 1, e^{i j \omega t}$ is bounded uniformly in $\operatorname{Im}(\omega) \geq 0$ and $t \geq 0$, so that $Z_{j}(t, \omega) \in \operatorname{Exp}_{+}(0)$ for any $t \geq 0$. On the other hand, when $j<0, \quad e^{i j \omega t}$ diverges as $\left|e^{i j \omega t}\right| \sim O\left(e^{|j t| \cdot \operatorname{Im}(\omega)}\right)$, so that $Z_{j}(t, \omega) \notin \operatorname{Exp}_{+}(0)$. Hence, if $i\left(Z_{j}(t)\right)=\left|Z_{j}\right\rangle$ converges to zero as $t \rightarrow \infty, \Pi_{c}\left|Z_{j}\right\rangle$ tends to zero when $j \geq 1$, although it may not tend to zero when $j<0$. In the usual center manifold theory, the projection to a center subspace is assumed to be continuous. Because of the discontinuity of $\Pi_{c}$, an interesting bifurcation occurs when $f(\theta) \neq \sin \theta$. In what follows, assume that $g$ is an even and unimodal function. In this case, on the imaginary axis, $T_{1}$ has only one resonance pole $\lambda_{0}=0$ at $K=K_{c}$. Hence,

$$
\mathbf{E}_{c}=\cdots \times\{0\} \times\{0\} \times \operatorname{span}\left\{\mu_{0}\right\} \times\{0\} \times\{0\} \times \cdots \subset \prod_{j \neq 0} X_{j}^{\prime}
$$

is of one dimension, where $\mu_{0}$ is the generalized eigenfunction associated with $\lambda_{0}=0$, which is given by

$$
\left\langle\phi \mid \mu_{0}\right\rangle:=\lim _{\lambda \rightarrow+0} \int_{\mathbf{R}} \frac{1}{\lambda-i \omega} \overline{\phi(\omega)} g(\omega) d \omega .
$$

This is also written as

$$
\left|\mu_{0}\right\rangle=\lim _{\lambda \rightarrow+0}\left|\frac{1}{\lambda-i \omega}\right\rangle,
$$

where the limit is taken with respect to the weak topology. The complementary subspace $\mathbf{E}_{c}^{\perp}$ of $\mathbf{E}_{c}$ in $\prod_{j \neq 0} X_{j}^{\prime}$ is the stable subspace. Next, let us derive the dynamics on a center manifold. The derivation is performed in the same way for both (a) a Gaussian distribution and (b) rational functions.

(I) First, we consider $f(\theta)=\sin \theta$. In this case, Eq. (10) is reduced to

$$
\frac{d}{d t}\left|Z_{1}\right\rangle=T_{1}^{\times}\left|Z_{1}\right\rangle-\frac{K}{2}\left\langle\overline{P_{0} \mid Z_{1}}\right\rangle\left|Z_{2}\right\rangle,
$$

and

$$
\frac{d}{d t}\left|Z_{j}\right\rangle=T_{j}^{\times}\left|Z_{j}\right\rangle-\frac{j K}{2}\left(\left\langle\overline{P_{0} \mid Z_{1}}\right\rangle\left|Z_{j+1}\right\rangle-\left\langle P_{0} \mid Z_{1}\right\rangle\left|Z_{j-1}\right\rangle\right),
$$

for $j=2,3, \ldots$. It is remarkable that these equations of $\left|Z_{1}\right\rangle,\left|Z_{2}\right\rangle, \ldots$ are independent of $\left|Z_{-1}\right\rangle,\left|Z_{-2}\right\rangle, \ldots$. Therefore, the projection $\Pi_{c}$ continuously acts on these equations. In order to investigate a bifurcation occurred at $K=K_{c}$, set $\varepsilon=K-K_{c}$. Then, Eq. (18) is rewritten as

$$
\frac{d}{d t}\left|Z_{1}\right\rangle=T_{10}^{\times}\left|Z_{1}\right\rangle+\frac{\varepsilon}{2}\left\langle P_{0} \mid Z_{1}\right\rangle\left|P_{0}\right\rangle-\frac{K}{2}\left\langle\overline{P_{0} \mid Z_{1}}\right\rangle\left|Z_{2}\right\rangle,
$$

where $T_{10}$ is defined by

$$
T_{10} \phi=i \omega \phi+\frac{K_{c}}{2}\left(P_{0}, \phi\right) P_{0},
$$

and $T_{10}^{\times}$is its dual operator. Note that $T_{10}$ has a resonance pole $\lambda_{0}=0$. In order to obtain the dynamics on the center manifold, we decompose a solution as

$$
\left|Z_{1}\right\rangle=\frac{K_{c}}{2} \alpha\left|\mu_{0}\right\rangle+\left|Y_{1}\right\rangle,
$$

along the direct sum $\mathbf{E}_{c} \oplus \mathbf{E}_{c}^{\perp}$. The $\alpha$ represents the coordinate on the center subspace, which is assumed to be sufficiently small; $|\alpha| \ll 1$. The purpose here is to derive the dynamics of $\alpha$. Since $\left|Y_{1}\right\rangle$ and $\left|Z_{2}\right\rangle$ are included in the stable subspace, they are higher order terms with respect to $\alpha ;\left|Y_{1}\right\rangle,\left|Z_{2}\right\rangle \sim O\left(\alpha^{2}\right)$.

Due to the definitions of $\mu_{0}$ and the resonance pole, we obtain $\left\langle P_{0} \mid \mu_{0}\right\rangle=2 / K_{c}$. This provides

$$
\left\langle P_{0} \mid Z_{1}\right\rangle=\alpha(t)+\left\langle P_{0} \mid Y_{1}\right\rangle .
$$

The projection of $\left|P_{0}\right\rangle$ is given by

$$
\Pi_{c}\left|P_{0}\right\rangle=\frac{K_{c}}{2 D_{0}}\left\langle P_{0} \mid \mu_{0}\right\rangle \cdot\left|\mu_{0}\right\rangle=\frac{1}{D_{0}}\left|\mu_{0}\right\rangle .
$$

Hence, $\left|P_{0}\right\rangle$ is decomposed as

$$
\left|P_{0}\right\rangle=\frac{1}{D_{0}}\left|\mu_{0}\right\rangle+\left|Y_{0}\right\rangle,
$$

where $\left|Y_{0}\right\rangle \in \mathbf{E}_{c}^{\perp}$ and $\left|Y_{0}\right\rangle \sim O\left(\alpha^{2}\right)$. Substituting Eqs. (21) and (22) into Eq. (19) for $j=2$ yields 


$$
\begin{aligned}
\frac{d}{d t}\left|Z_{2}\right\rangle= & T_{2}^{\times}\left|Z_{2}\right\rangle-K\left(\left(\bar{\alpha}+\left\langle\overline{P_{0} \mid Y_{1}}\right\rangle\right) \cdot\left|Z_{3}\right\rangle\right. \\
& \left.-\left(\alpha+\left\langle P_{0} \mid Y_{1}\right\rangle\right)\left(\frac{K_{c}}{2} \alpha\left|\mu_{0}\right\rangle+\left|Y_{1}\right\rangle\right)\right) .
\end{aligned}
$$

We suppose that $d \alpha / d t \sim O\left(\alpha^{2}, \alpha \varepsilon, \varepsilon^{2}\right)$. Then, the above equation yields

$$
T_{2}^{\times}\left|Z_{2}\right\rangle=-\frac{K K_{c}}{2} \alpha^{2}\left|\mu_{0}\right\rangle+O\left(\alpha^{3}, \alpha^{2} \varepsilon, \alpha \varepsilon^{2}, \varepsilon^{3}\right) .
$$

Lemma 1. Define the operator $\left(T_{2}^{\times}\right)^{-1}: X \rightarrow X^{\prime}$ to be

$$
\left\langle\phi \mid\left(T_{2}^{\times}\right)^{-1} \psi\right\rangle=-\frac{1}{2} \lim _{x \rightarrow+0} \int_{\mathbf{R}} \frac{1}{x-i \omega} \overline{\phi(\omega)} \psi(\omega) g(\omega) d \omega .
$$

Then,

$$
\left(T_{2}^{\times}\right)\left(T_{2}^{\times}\right)^{-1}|\psi\rangle=\left(T_{2}^{\times}\right)^{-1}\left(T_{2}^{\times}\right)|\psi\rangle=|\psi\rangle
$$

for any $\psi \in X$.

Proof. Note that $T_{2} \phi=2 i \omega \phi$ when $f(\theta)=\sin \theta$. A straightforward calculation shows that

$$
\begin{aligned}
\left\langle\phi \mid\left(T_{2}^{\times}\right)\left(T_{2}^{\times}\right)^{-1} \psi\right\rangle= & \left\langle T_{2}^{*} \phi \mid\left(T_{2}^{\times}\right)^{-1} \psi\right\rangle \\
= & -\frac{1}{2} \lim _{x \rightarrow+0} \int_{\mathbf{R}} \frac{2 i \omega}{\overline{x-i \omega} \overline{\phi(\omega)} \psi(\omega) g(\omega) d \omega} \\
= & \int_{\mathbf{R}} \overline{\phi(\omega)} \psi(\omega) g(\omega) d \omega \\
& -\lim _{x \rightarrow+0} \int_{\mathbf{R}} \frac{x}{x-i \omega} \overline{\phi(\omega)} \psi(\omega) g(\omega) d \omega \\
= & \int_{\mathbf{R}} \overline{\phi(\omega)} \psi(\omega) g(\omega) d \omega .
\end{aligned}
$$

Thus, we obtain

$$
\left\langle\phi \mid\left(T_{2}^{\times}\right)\left(T_{2}^{\times}\right)^{-1} \psi\right\rangle=\langle\phi \mid \psi\rangle,
$$

for any $\phi \in X$. The latter equality $\left\langle\phi \mid\left(T_{2}^{\times}\right)^{-1}\left(T_{2}^{\times}\right) \psi\right\rangle$ $=\langle\phi \mid \psi\rangle$ is proved in the same way.

Using the definition of $\mu_{0},\left(T_{2}^{\times}\right)^{-1}\left|\mu_{0}\right\rangle$ is calculated as

$$
\begin{aligned}
\left\langle\phi \mid\left(T_{2}^{\times}\right)^{-1} \mu_{0}\right\rangle & =\lim _{x \rightarrow+0}\left\langle\phi \mid\left(T_{2}^{\times}\right)^{-1} \frac{1}{x-i \omega}\right\rangle \\
& =-\frac{1}{2} \lim _{x \rightarrow+0} \int_{\mathbf{R}} \frac{1}{(x-i \omega)^{2}} \overline{\phi(\omega)} g(\omega) d \omega .
\end{aligned}
$$

Then, Eq. (25) provides

$$
\begin{aligned}
\left\langle\phi \mid Z_{2}\right\rangle= & \frac{K K_{c}}{4} \alpha^{2} \lim _{x \rightarrow+0} \int_{\mathbf{R}} \frac{1}{(x-i \omega)^{2}} \overline{\phi(\omega)} g(\omega) d \omega \\
& +O\left(\alpha^{3}, \alpha^{2} \varepsilon, \alpha \varepsilon^{2}, \varepsilon^{3}\right),
\end{aligned}
$$

which gives the expression of the center manifold to the $\left|Z_{2}\right\rangle$ direction. The projection of it to the center subspace is given as

$$
\begin{aligned}
\Pi_{c}\left|Z_{2}\right\rangle & =\frac{K_{c}}{2 D_{0}}\left\langle\overline{Z_{2}} \mid \mu_{0}\right\rangle \cdot\left|\mu_{0}\right\rangle \\
& =\frac{K_{c}}{2 D_{0}} \lim _{x \rightarrow+0}\left\langle\overline{Z_{2}} \mid \frac{1}{x-i \omega}\right\rangle \cdot\left|\mu_{0}\right\rangle \\
& =\frac{K_{c}}{2 D_{0}} \lim _{x \rightarrow+0}\left\langle\frac{1}{x+i \omega} \mid Z_{2}\right\rangle \cdot\left|\mu_{0}\right\rangle .
\end{aligned}
$$

In what follows, higher order terms with respect to $\alpha$ and $\varepsilon$ are denoted by h.o.t. Equation (28) yields

$$
\begin{aligned}
\lim _{x \rightarrow+0} & \left\langle\frac{1}{x+i \omega} \mid Z_{2}\right\rangle \\
& =\frac{K K_{c}}{4} \alpha^{2} \lim _{x \rightarrow+0} \int_{\mathbf{R}} \frac{1}{(x-i \omega)^{3}} g(\omega) d \omega+\text { h.o.t. } \\
& =-\frac{K K_{c}}{8} \alpha^{2} \lim _{x \rightarrow+0} \int_{\mathbf{R}} \frac{1}{x-i \omega} g^{\prime \prime}(\omega) d \omega+\text { h.o.t. } \\
& =-\frac{K K_{c}}{8} \alpha^{2} \cdot \pi g^{\prime \prime}(0)+\text { h.o.t. }
\end{aligned}
$$

For the last equality, we used a formula of the Poisson kernel. Thus, we obtain

$$
\Pi_{c}\left|Z_{2}\right\rangle=-\frac{K K_{c}^{2}}{16 D_{0}} \alpha^{2} \cdot \pi g^{\prime \prime}(0) \cdot\left|\mu_{0}\right\rangle+\text { h.o.t. }
$$

Finally, the projection of Eq. (20) to the center subspace is given by

$$
\begin{aligned}
\frac{d}{d t} \Pi_{c}\left|Z_{1}\right\rangle= & \Pi_{c} T_{10}^{\times}\left|Z_{1}\right\rangle+\frac{\varepsilon}{2}\left\langle P_{0} \mid Z_{1}\right\rangle \Pi_{c}\left|P_{0}\right\rangle \\
& -\frac{K}{2}\left\langle\overline{P_{0} \mid Z_{1}}\right\rangle \Pi_{c}\left|Z_{2}\right\rangle .
\end{aligned}
$$

Recall that a resonance pole is an eigenvalue of the dual operator. Since $\Pi_{c}$ is a projection to the eigenspace associated with a resonance pole $\lambda_{0}=0$ of $T_{10}^{\times}$, we have

$$
\Pi_{c} T_{10}^{\times}=T_{10}^{\times} \Pi_{c}=0 .
$$

By using Eqs. (21)-(23), (29), and (30), we obtain

$$
\begin{aligned}
\frac{d}{d t} & \frac{K_{c}}{2} \alpha\left|\mu_{0}\right\rangle \\
= & \frac{\varepsilon}{2}\left(\alpha+\left\langle P_{0} \mid Y_{1}\right\rangle\right) \frac{1}{D_{0}}\left|\mu_{0}\right\rangle \\
& \quad-\frac{K}{2}\left(\bar{\alpha}+\left\langle\overline{P_{0} \mid Y_{1}}\right\rangle\right) \cdot\left(-\frac{\pi g^{\prime \prime}(0) K K_{c}^{2}}{16 D_{0}} \alpha^{2}\left|\mu_{0}\right\rangle+\text { h.o.t. }\right), \\
= & \frac{\varepsilon}{2 D_{0}} \alpha\left|\mu_{0}\right\rangle+\frac{\pi g^{\prime \prime}(0) K_{c}^{4}}{32 D_{0}} \alpha|\alpha|^{2}\left|\mu_{0}\right\rangle+\text { h.o.t., }
\end{aligned}
$$

which yields the dynamics on the center manifold as

$$
\frac{d}{d t} \alpha=\frac{\alpha}{D_{0} K_{c}}\left(\varepsilon+\frac{\pi g^{\prime \prime}(0) K_{c}^{4}}{16}|\alpha|^{2}\right)+\text { h.o.t. }
$$

Since $g^{\prime \prime}(0)<0$ for the Gaussian distribution, this equation has a fixed point expressed as

$$
\alpha=\sqrt{\frac{-16}{\pi K_{c}^{4} g^{\prime \prime}(0)}} \sqrt{K-K_{c}}+O\left(K-K_{c}\right),
$$

when $\varepsilon=K-K_{c}>0$. Note that Kuramoto's order parameter $\eta_{1}(t)=\left(P_{0}, Z_{1}\right)$ is given as

$$
\eta_{1}(t)=\left\langle P_{0} \mid Z_{1}\right\rangle=\alpha+\text { h.o.t. }
$$

Thus, the dynamics of the order parameter is also given by Eq. (31). To prove that the fixed point (32) is asymptotically stable, it is sufficient to show the following. 
Lemma 2. $D_{0}>0$.

Proof. This is proved by using the definition of $D_{0}$

$$
D_{0}=\lim _{\lambda \rightarrow 0} \frac{1}{\lambda}\left(1-\frac{K_{c}}{2} \int_{\mathbf{R}} \frac{g(\omega)}{\lambda-i \omega} d \omega-\pi K_{c} g(-i \lambda)\right)
$$

and the fact that $g(\omega)$ is an even and unimodal function. See Ref. 13 for the details.

Since $D_{0}>0, K_{c}>0, g^{\prime \prime}(0)<0$, the fixed point $\alpha=0$ (de-synchronous state) is unstable and the fixed point Eq. (32) (synchronous state) is asymptotically stable when $\varepsilon=K-K_{c}>0$. This confirms the Kuramoto's bifurcation diagram.

(II) Assume that $f(\theta)=\sin \theta+h \sin 2 \theta$ with $h \in \boldsymbol{R}$. Then, Eq. (10) is reduced to

$$
\begin{aligned}
\frac{d}{d t}\left|Z_{1}\right\rangle= & T_{10}^{\times}\left|Z_{1}\right\rangle+\frac{\varepsilon}{2}\left\langle P_{0} \mid Z_{1}\right\rangle\left|P_{0}\right\rangle \\
& -\frac{K}{2}\left(\left\langle\overline{P_{0} \mid Z_{1}}\right\rangle\left|Z_{2}\right\rangle+h\left\langle\overline{P_{0} \mid Z_{2}}\right\rangle\left|Z_{3}\right\rangle\right. \\
& \left.-h\left\langle P_{0} \mid Z_{2}\right\rangle\left|Z_{-1}\right\rangle\right),
\end{aligned}
$$

and

$$
\begin{aligned}
\frac{d}{d t}\left|Z_{2}\right\rangle= & T_{2}^{\times}\left|Z_{2}\right\rangle-K\left(\left\langle\overline{P_{0} \mid Z_{1}}\right\rangle\left|Z_{3}\right\rangle\right. \\
& \left.+h\left\langle\overline{P_{0} \mid Z_{2}}\right\rangle\left|Z_{4}\right\rangle-\left\langle P_{0} \mid Z_{1}\right\rangle\left|Z_{1}\right\rangle\right),
\end{aligned}
$$

for $j=1,2$, respectively, where

$$
T_{2} \phi=2 i \omega \phi+K h\left(P_{0}, \phi\right) P_{0} .
$$

Note that Eq. (34) includes $\left|Z_{-1}\right\rangle$, on which $\Pi_{c}$ is a discontinuous operator. Equation (35) provides

$$
T_{2}^{\times}\left|Z_{2}\right\rangle=-\frac{K K_{c}}{2} \alpha^{2}\left|\mu_{0}\right\rangle+\text { h.o.t. }
$$

as before.

Lemma 3. Define the operator $\left(T_{2}^{\times}\right)^{-1}: X \rightarrow X^{\prime}$ to be

$$
\begin{aligned}
\left\langle\phi \mid\left(T_{2}^{\times}\right)^{-1} \psi\right\rangle= & -\frac{1}{2} \lim _{x \rightarrow+0} \int_{\mathbf{R}} \frac{1}{x-i \omega} \overline{\phi(\omega)} \psi(\omega) g(\omega) d \omega \\
& -\frac{K h}{2} \frac{1}{2-h K \pi g(0)} \\
& \times \lim _{x \rightarrow+0} \int_{\mathbf{R}} \frac{\overline{\phi(\omega)} g(\omega)}{x-i \omega} d \omega \cdot \int_{\mathbf{R}} \frac{\psi(\omega) g(\omega)}{x-i \omega} d \omega .
\end{aligned}
$$

Then,

$$
\left(T_{2}^{\times}\right)\left(T_{2}^{\times}\right)^{-1}|\psi\rangle=\left(T_{2}^{\times}\right)^{-1}\left(T_{2}^{\times}\right)|\psi\rangle=|\psi\rangle
$$

for any $\psi \in X$.

This lemma is verified by a straightforward calculation as the proof of Lemma 1. This is applied to Eq. (37) to yield

$$
\begin{aligned}
\left\langle\phi \mid Z_{2}\right\rangle= & -\frac{K K_{c}}{2} \alpha^{2}\left\langle\phi \mid\left(T_{2}^{\times}\right)^{-1} \mu_{0}\right\rangle+\text { h.o.t. } \\
= & \frac{K_{c}^{2}}{4} \alpha^{2} \lim _{x \rightarrow+0} \int_{\mathbf{R}} \frac{1}{(x-i \omega)^{2}} \overline{\phi(\omega)} g(\omega) d \omega \\
& +\frac{1}{4} \frac{K_{c}^{3} h \alpha^{2}}{2-h K_{c} \pi g(0)} \lim _{x \rightarrow+0} \int_{\mathbf{R}} \frac{\overline{\phi(\omega)} g(\omega)}{x-i \omega} d \omega \\
& \times \int_{\mathbf{R}} \frac{g(\omega)}{(x-i \omega)^{2}} d \omega+\text { h.o.t. }
\end{aligned}
$$

Define the negative constant $C$ to be

$$
C=-\lim _{x \rightarrow+0} \int_{\mathbf{R}} \frac{g(\omega)}{(x-i \omega)^{2}} d \omega=\text { p.v. } \int_{\mathbf{R}} \frac{g^{\prime}(\omega)}{\omega} d \omega .
$$

Since $g(\omega)$ is assumed to be even and unimodal, Eq. (8) provides

$$
K_{c}=K_{c}^{(1)}=\frac{2}{\pi g(0)}
$$

Hence, we obtain

$$
\begin{aligned}
\left\langle\phi \mid Z_{2}\right\rangle= & \frac{K_{c}^{2}}{4} \alpha^{2} \lim _{x \rightarrow+0} \int_{\mathbf{R}} \frac{1}{(x-i \omega)^{2}} \overline{\phi(\omega)} g(\omega) d \omega \\
& -\frac{K_{c}^{3} C h \alpha^{2}}{8(1-h)} \lim _{x \rightarrow+0} \int_{\mathbf{R}} \frac{\overline{\phi(\omega)} g(\omega)}{x-i \omega} d \omega+\text { h.o.t. }
\end{aligned}
$$

In particular,

$$
\begin{aligned}
\left\langle P_{0} \mid Z_{2}\right\rangle & =-\frac{K_{c}^{2}}{4} \alpha^{2} C-\frac{K_{c}^{3} C h \alpha^{2}}{8(1-h)} \pi g(0)+\text { h.o.t. } \\
& =-\frac{K_{c}^{2} C}{4(1-h)} \alpha^{2}+\text { h.o.t. }
\end{aligned}
$$

Since $\left|Z_{2}\right\rangle \sim O\left(\alpha^{2}\right)$ and $\Pi_{c}$ continuously acts on $\left|Z_{2}\right\rangle, \Pi_{c}\left|Z_{2}\right\rangle$ is also of order $\alpha^{2}$. Similarly, we have $\Pi_{c}\left|Z_{3}\right\rangle \sim O\left(\alpha^{2}\right)$. Hence,

$$
\left\langle\overline{P_{0} \mid Z_{1}}\right\rangle\left|Z_{2}\right\rangle \sim O\left(\alpha^{3}\right), \quad\left\langle\overline{P_{0} \mid Z_{2}}\right\rangle\left|Z_{3}\right\rangle \sim O\left(\alpha^{3}\right) .
$$

Substituting Eqs. (21)-(23), (30), (41), and (42) into Eq. (34), we obtain

$$
\frac{d}{d t} \frac{K_{c}}{2} \alpha\left|\mu_{0}\right\rangle=\frac{\varepsilon \alpha}{2 D_{0}}\left|\mu_{0}\right\rangle-\frac{K_{c} h}{2} \frac{K_{c}^{2} C}{4(1-h)} \alpha^{2} \cdot \Pi_{c}\left|Z_{-1}\right\rangle+\text { h.o.t. }
$$

The final task is to calculate $\Pi_{c}\left|Z_{-1}\right\rangle$. Since $\Pi_{c}$ is discontinuous at $\left|Z_{-1}\right\rangle$, it is expected that $\Pi_{c}\left|Z_{-1}\right\rangle \sim O(1)$ even if $\left|Z_{-1}\right\rangle \sim O(\alpha)$. In this manner, the quadratic nonlinearity appears in Eq. (43), although the cubic nonlinearity was dominant in the case (I).

Lemma $4 . \Pi_{c}\left|Z_{-1}\right\rangle$ is given by

$$
\Pi_{c}\left|Z_{-1}\right\rangle=\frac{2}{D_{0}} e^{-i \arg (\alpha)}+O(\alpha) .
$$

This will be proved in Appendix. Then, we obtain the dynamics on the center manifold as 

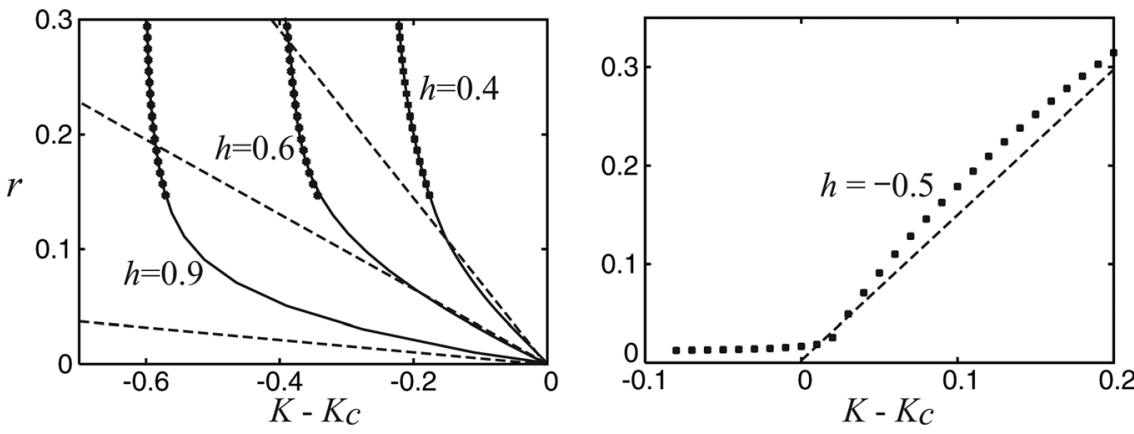

FIG. 4. Numerical results for $f(\theta)=\sin \theta+h \sin 2 \theta$. Black dots denote the order parameter calculated from Eq. (1) for $N=8000, g(\omega)=e^{-\omega^{2} / 2} / \sqrt{2 \pi}$ using the method shown in Ref. 18. Since $r$ is unstable when $K-K_{c}<0$, it is difficult to obtain small $r$. The solid lines are interpolations of black dots using quintic polynomials. The dotted lines denote the analytical results obtained by Eq. (46).

$$
\frac{d \alpha}{d t}=\frac{\alpha}{D_{0} K_{c}}\left(\varepsilon-\frac{K_{c}^{3} C h}{2(1-h)} \alpha e^{-i \arg (\alpha)}\right)+\text { h.o.t. }
$$

which proves that there exists a fixed point that is expressed as

$$
r=\left|\eta_{1}\right| \sim|\alpha|=\frac{2(1-h)}{K_{c}^{3} C h}\left(K-K_{c}\right)+O\left(\left(K-K_{c}\right)^{2}\right) .
$$

Since $C<0$, for $h<0$, a stable branch emerges when $K_{c}<K$, and for $0<h<1$, an unstable branch emerges when $K<K_{c}$ (Fig. 1(b)). (The case $h>1$ does not satisfy the assumption $\inf K_{c}^{(j)}=K_{c}^{(1)}$ ).

\section{DISCUSSION}

Equation (10) shows that the dynamics of $Z_{1}, Z_{2}, \ldots$ are independent of $Z_{-1}, Z_{-2}, \ldots$ if and only if $f(\theta)=\sin \theta$. In other words, Eq. (10) splits into two systems: a system of $\left\{Z_{1}, Z_{2}, \ldots\right\}$ and a system of $\left\{Z_{-1}, Z_{-2}, \ldots\right\}$. Since the projection $\Pi_{c}$ is continuous on a solution of the former system, we can show the existence of a smooth center manifold. Note that Eq. (1) is invariant under the rotation on a circle. As a result, the dynamics on the center manifold is also invariant under the rotation $\alpha \mapsto e^{i \phi} \alpha$. If a center manifold is smooth, then the dynamics on this manifold with the rotation symmetry must be of the form $\dot{\alpha}=\alpha F\left(|\alpha|^{2}\right)$. Thus, a cubic nonlinearity is dominant, and a pitchfork bifurcation generally occurs, as shown in Eq. (31). On the other hand, if $f(\theta) \neq \sin \theta$, then the equations of $Z_{1}, Z_{2}, \ldots$ depend on $Z_{-1}, Z_{-2}, \ldots$, on which $\Pi_{c}$ is not continuous. In such a case, the center manifold is not smooth, and quadratic nonlinearity may appear, as described above. In this manner, different bifurcations occur when $f(\theta) \neq \sin \theta$. Although the diagram shown in Fig. 1(b) looks like a transcritical bifurcation, Eq. (45) is different from the normal form of a transcritical bifurcation. Because of the factor $e^{-i \arg (\alpha)}$ caused by the discontinuity of $\Pi_{c}$, Eq. (45) remains invariant under the rotation despite the existence of a quadratic nonlinearity. The discontinuity induces a new type of bifurcation including $e^{-i \arg (\alpha)}$.

A center manifold reduction for globally coupled phase oscillators was also developed by Crawford and Davies 9 with noise of strength $D>0$. Although they also expected a diagram such as shown as Fig. 1(b) when $D=0$, the factor $e^{-i \arg (\alpha)}$ was not obtained. Since the eigenfunction diverges as $D \rightarrow 0$, expressions of the dynamics on the center manifold were not shown explicitly. In the present paper, we have shown that the eigenfunction $\mu_{0}$ exists on a space of generalized functions, which provides a correct center manifold reduction. The diagram shown in Fig. 1(b) was also obtained by Daido ${ }^{4}$ by means of a self-consistent analysis. Unfortunately, his results were not correct because he performed inappropriate termwise integrations of certain infinite series. According to his results, the order parameter for $f(\theta)=\sin \theta$ $+h \sin 2 \theta$ is given as $(1-2 h) \cdot$ const., which suggests that some degeneracy occurs when $h=1 / 2$. However, the numerical results given in Fig. 4 show that the critical exponent of the order parameter changes only when $h=0,1$, which agrees with the results of the present study (46). Ott and Antonsen ${ }^{10}$ found an inertia manifold given by $Z_{n}=\left(Z_{1}\right)^{n}$ when $f(\theta)=\sin \theta$ (see also Marvel et al. ${ }^{11}$ ). The center manifold of the present study is a finite-dimensional submanifold of the inertia manifold, which provides a further reduction of the results of Ott and Antonsen ${ }^{10}$ and Pikovsky and Rosenblum. ${ }^{12}$ Furthermore, our method is independent of the choice of the coupling function $f$ and the distribution $g(\omega)$, although, in the literature, certain specific functions $f$ inducing symmetries and the Lorentzian distribution were mainly considered. The advantage of Ott and Antonsen's reduction based on the symmetry is that their results are valid on the whole phase space and for any $K$, while our center manifold reduction is a local theory that is applicable near the fixed state and near the transition point $K_{c}$.

In order to apply our center manifold reduction, the initial conditions for $Z_{j}(t, \omega)$ are assumed to be included in the test functions space $X$ so that the decomposition (21) holds (the first term of Eq. (21) is obtained as $\Pi_{c}\left|Z_{1}\right\rangle$ and the domain of the projection is $X$ ). In particular, the initial condition $\rho_{0}(\theta, \omega)=h(\theta, \omega)$ for the system (3) is analytic in $\omega$ on the upper half plane because $X$ consists of analytic functions on the upper half plane. In most literature, $h(\theta, \omega)=h(\theta)$ is assumed to be independent of $\omega$ and thus this assumption is satisfied. When the initial condition $h(\theta, \omega)$ or the distribution $g(\omega)$ is not analytic, our theory is not applicable. In such a case, the order parameter may decay with a polynomial rate as $t$ increases even when $K<K_{c}$, see Ref. 8. To establish a mathematical theory of bifurcation for such a polynomial decay is a challenging task.

The key strategy of the present theory is to use spaces of generalized functions and the weak topology. The weak topology is suitable for investigating the dynamics of moments of probability density functions. Such a study using a space of generalized functions is systematically formulated by means of a rigged Hilbert space, on which both of a Hilbert 
space and a space of generalized functions are available. Since the strategy is independent of the details of the models, this strategy will be extended to various types of large populations of coupled systems and evolution equations of density functions, such as the Vlasov equation.

\section{ACKNOWLEDGMENTS}

The present study was supported by Grant-in-Aid for Young Scientists (B), No.22740069 from MEXT Japan.

\section{APPENDIX}

In this appendix, we prove Lemma 4 . By the definition of $\Pi_{c}$,

$$
\Pi_{c}\left|Z_{-1}\right\rangle=\frac{K_{c}}{2 D_{0}}\left\langle\bar{Z}_{-1} \mid \mu_{0}\right\rangle\left|\mu_{0}\right\rangle=\frac{K_{c}}{2 D_{0}}\left\langle Z_{1} \mid \mu_{0}\right\rangle\left|\mu_{0}\right\rangle .
$$

First, let us calculate $\left\langle Z_{1}+\bar{Z}_{1} \mid \mu_{0}\right\rangle$ as

$$
\begin{aligned}
\left\langle Z_{1}+\bar{Z}_{1} \mid \mu_{0}\right\rangle= & \lim _{x \rightarrow+0} \int_{\mathbf{R}} \frac{1}{x-i \omega}\left(Z_{1}(t, \omega)+\overline{Z_{1}(t, \omega)}\right) g(\omega) d \omega \\
= & \lim _{x \rightarrow+0} \int_{\mathbf{R}} \frac{x}{x^{2}+\omega^{2}}\left(Z_{1}(t, \omega)+\overline{Z_{1}(t, \omega)}\right) g(\omega) d \omega \\
& +i \lim _{x \rightarrow+0} \int_{\mathbf{R}} \frac{\omega}{x^{2}+\omega^{2}}\left(Z_{1}(t, \omega)+\overline{Z_{1}(t, \omega)}\right) g(\omega) d \omega \\
= & \pi g(0)\left(Z_{1}(t, 0)+\overline{Z_{1}(t, 0)}\right)+i q(t),
\end{aligned}
$$

where

$$
q(t)=\lim _{x \rightarrow+0} \int_{\mathbf{R}} \frac{\omega}{x^{2}+\omega^{2}}\left(Z_{1}(t, \omega)+\overline{Z_{1}(t, \omega)}\right) g(\omega) d \omega
$$

is a real-valued function. The next task is to estimate $Z_{1}(t, 0)$. It is well known that the continuous model (3) can be integrated along the characteristic curve. The characteristic curve is defined as a solution of the equation

$$
\frac{d x}{d t}=\omega+K \sum_{l=-\infty}^{\infty} f_{l} \eta_{l}(t) e^{-i l x}
$$

When $f(\theta)=\sin \theta+h \sin 2 \theta$, this becomes

$$
\frac{d x}{d t}=\omega+\frac{K}{2 i}\left(\eta_{1} e^{-i x}-\bar{\eta}_{1} e^{i x}\right)+\frac{K h}{2 i}\left(\eta_{2} e^{-2 i x}-\bar{\eta}_{2} e^{2 i x}\right) .
$$

Since $\eta_{1}(t)=\alpha+O\left(\alpha^{2}\right)$ and $\eta_{2}(t)=\left\langle P_{0} \mid Z_{2}\right\rangle \sim O\left(\alpha^{2}\right)$ on the center manifold (see Eq. (33)),

$$
\frac{d x}{d t}=\omega+K|\alpha| \sin (\arg (\alpha)-x)+O\left(\alpha^{2}\right) .
$$

Let $x=x(t, s ; \theta, \omega)$ be a solution of this equation satisfying the initial condition $x(s, s ; \theta, \omega)=\theta$ at an initial time $s$. By integrating Eq. (3) along the characteristic curve and substituting the resultant in the definition of $Z_{k}(t, \omega)$, we can verify that $Z_{k}(t, \omega)$ is rewritten as

$$
Z_{k}(t, \omega)=\int_{0}^{2 \pi} e^{i k x(t, 0 ; \theta, \omega)} h(\theta, \omega) d \theta,
$$

where $h(\theta, \omega)=\rho_{0}(t, \omega)$ is the initial condition for $\rho_{t}$. To obtain $Z_{1}(t, 0)$, let us estimate $x(t, 0 ; \theta, 0)$, which is a solution of the equation

$$
\frac{d x}{d t}=K|\alpha| \sin (\arg (\alpha)-x)+O\left(\alpha^{2}\right) .
$$

This equation has a stable fixed point $x=\arg (\alpha)+O(\alpha)$. Hence, we obtain

$$
Z_{1}(t, 0)=\int_{0}^{2 \pi} e^{i(\arg (\alpha)+O(\alpha))} h(\theta, \omega) d \theta=e^{i \arg (\alpha)}+O(\alpha)
$$

when $t$ is sufficiently large. Substituting it into Eq. (A2) yields

$$
\left\langle Z_{1}+\bar{Z}_{1} \mid \mu_{0}\right\rangle=2 \pi g(0) \cos (\arg (\alpha))+i q(t)+O(\alpha) .
$$

Since

$$
\Pi_{c}\left|Z_{1}\right\rangle=\frac{K_{c}}{2 D_{0}}\left\langle\bar{Z}_{1} \mid \mu_{0}\right\rangle\left|\mu_{0}\right\rangle=\frac{K_{c}}{2} \alpha\left|\mu_{0}\right\rangle \sim O(\alpha),
$$

we have

$$
\left\langle Z_{1} \mid \mu_{0}\right\rangle=2 \pi g(0) \cos (\arg (\alpha))+i q(t)+O(\alpha) .
$$

Now $q(t)$ is determined by the symmetry of the system. Note that Eq. (10) is invariant under the rotation $Z_{j} \mapsto e^{i j \phi} Z_{j}$. This symmetry arises from the rotation symmetry $\theta \mapsto \theta+\phi$ of Eq. (1). It follows from Eq. (A6) that $\alpha$ is also mapped to $e^{i \phi} \alpha$ by this transformation. Thus Eq. (A7) has to be invariant under the transformation $Z_{1} \mapsto e^{i \phi} Z_{1}$ and $\arg (\alpha) \mapsto \arg (\alpha)+\phi$. Since $q(t)$ is real-valued, it turns out that it is of the form $q(t)=-2 \pi g(0) \sin (\arg (\alpha))+O(\alpha)$ and

$$
\left\langle Z_{1} \mid \mu_{0}\right\rangle=2 \pi g(0) e^{-i \arg (\alpha)}+O(\alpha)=\frac{4}{K_{c}} e^{-i \arg (\alpha)}+O(\alpha) .
$$

This and Eq. (A1) prove Lemma 4.

${ }^{1}$ A. Pikovsky, M. Rosenblum, and J. Kurths, Synchronization: A Universal Concept in Nonlinear Sciences (Cambridge University Press, Cambridge, 2001).

${ }^{2}$ Y. Kuramoto, Chemical Oscillations, Waves, and Turbulence (SpringerVerlag, Berlin, 1984).

${ }^{3}$ S. H. Strogatz, Physica D 143, 1 (2000).

${ }^{4}$ H. Daido, Physica D 91, 24 (1996).

${ }^{5}$ R. E. Mirollo and S. H. Strogatz, J. Stat. Phys. 60, 245 (1990).

${ }^{6}$ R. E. Mirollo and S. H. Strogatz, J. Nonlinear Sci. 17, 309 (2007).

${ }^{7}$ S. H. Strogatz and R. E. Mirollo, J. Stat. Phys. 63, 613 (1991).

${ }^{8}$ S. H. Strogatz, R. E. Mirollo, and P. C. Matthews, Phys. Rev. Lett. 68, 2730 (1992)

${ }^{9}$ J. D. Crawford and K. T. R. Davies, Physica D 125, 1 (1999).

${ }^{10}$ E. Ott and T. M. Antonsen, Chaos, 18, 037113 (2008).

${ }^{11}$ S. A. Marvel, R. E. Mirollo, and S. H. Strogatz, Chaos, 19, 043104 (2009).

${ }^{12}$ A. Pikovsky and M. Rosenblum, Phys. Rev. Lett. 101, 264103 (2008).

${ }^{13}$ H. Chiba, e-print arXiv: 1008.0249.

${ }^{14}$ N. J. Balmforth and R. Sassi, Physica D 143, 21 (2000).

${ }^{15}$ I. M. Gelfand and N. Y. Vilenkin, Generalized Functions (Springer-Verlag, New York, 1964), Vol. 4.

${ }^{16}$ A. Bohm and M. Gadella, Dirac kets, Gamow Vectors and Gelfand Triplets (Lecture Notes in Physics, 348, Springer-Verlag, Berlin, 1989).

${ }^{17}$ E. A. Martens, E. Barreto, S. H. Strogatz, E. Ott, P. So, and T. M. Antonsen, Phys. Rev. E 79, 026204 (2009).

${ }^{18}$ R. Tönjes, N. Masuda, and H. Kori, Chaos 20, 033108 (2010). 